\title{
Modélisation de coiffures naturelles à partir des propriétés physiques du cheveu
}

\author{
F. Bertails ${ }^{1}$, B. Audoly ${ }^{2}$, B. Querleux ${ }^{3}$, M.-P. Cani ${ }^{1}$, F. Leroy ${ }^{3}$ et J.-L. Lévêque ${ }^{3}$ \\ ${ }^{1}$ EVASION, Laboratoire GRAVIR-IMAG/INRIA, Grenoble \\ ${ }^{2}$ Laboratoire de Modélisation en Mécanique, Univ. Pierre et Marie Curie, Paris 6 \\ ${ }^{3}$ L'Oréal Recherche, Paris
}
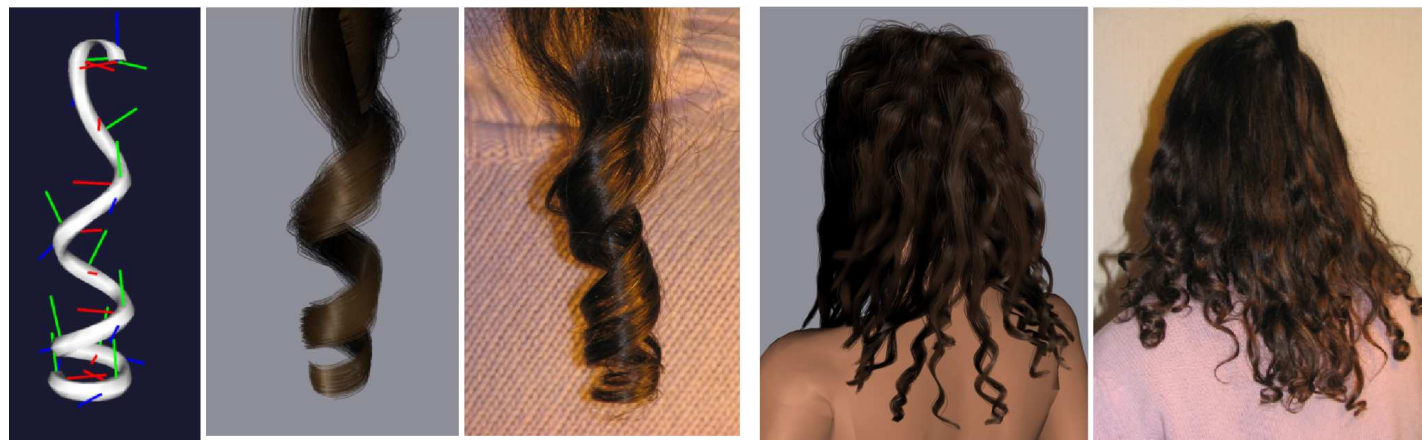

FIG. 1 - Cheveux bouclés réels vs. synthétiques. De gauche à droite : configuration à l'équilibre d'un cheveu bouclé; mèche générée de manière procédurale à partir de ce cheveu; mèche réelle dont on s'est inspiré pour la modélisation; chevelure complète composée d'une centaine de mèches; chevelure réelle dont on s'est inspiré pour la modélisation.

Résumé : Nous présentons une nouvelle approche physique permettant de simuler la forme d'une chevelure naturelle à partir des propriétés physiques, structurelles et ethniques caractérisant les cheveux d'une personne. Pour cela, nous nous basons sur un modèle mécanique connu pour les tiges élastiques - modèle de Kirchhoff - capable de prendre en compte la frisure naturelle d'un cheveu, ainsi que la géométrie elliptique de sa section. La forme à l'équilibre d'un cheveu est calculée de manière stable et efficace par la minimisation de son énergie potentielle. Cette technique, étendue à l'échelle de la mèche, nous permet de générer diverses configurations typiques telles que les boucles anglaises. Comme le montrent nos résultats, la méthode est capable de prédire la forme au naturel d'une chevelure, quel que soit le type ethnique d'origine, et à partir d'un nombre réduit de paramètres. De plus, elle offre à l'utilisateur la possibilité de réaliser virtuellement des opérations de coiffure classiques telles que le mouillage, la coupe et le séchage.

Mots-clés : Chevelure, simulation physique, coiffure virtuelle

\section{Introduction et travaux antérieurs}

La chevelure est un élément visuel très important d'une personne humaine. Elle reflète notamment l'origine ethnique et influe bien souvent sur la perception que les autres ont de soi $^{1}$. Avec la multiplication, ces dernières années, des personnages de synthèse dans les environnements virtuels, la synthèse de chevelures virtuelles est rapidement apparue comme un problème important et difficile pour la modélisation réaliste de personnages.

Ainsi, beaucoup de travaux en informatique graphique se sont récemment penchés sur le problème de modélisation de coiffures virtuelles. Cependant, aucun modèle existant n'est capable de tenir compte précisément des propriétés structurelles du cheveu pour en déduire sa forme, ni de prédire à l'avance la forme que prendrait une chevelure donnée en poussant ou une fois mouillée. Offrir de telles possibilités pourrait par exemple permettre le développement d'un "salon de coiffure" virtuel, destiné aux usagers pour l'essayage de coupes virtuelles, ou même à la formation des apprentis coiffeurs. Le travail que nous présentons ici est, à notre connaissance, la première avancée dans cette voie.

\footnotetext{
${ }^{1}$ Selon des études intitulées "First Impressions and Hair Impressions", menées par M. Lafrance, professeur en psychologie à l'Université de Yale (http ://www.physique.com/ca_fre/sn/sn_yale-study2.asp)
} 


\subsection{Propriétés structurelles et mécaniques du cheveu}

Une bonne compréhension des propriétés géométriques et mécaniques du cheveu est nécessaire à l'élaboration d'un modèle réaliste. Un cheveu est un tube elliptique très fin et très léger, encastré dans le cuir chevelu, et qui se déforme de manière anisotrope : un cheveu se courbe facilement et peut éventuellement s'enrouler autour de luimême, mais il est pratiquement inextensible et peu soumis aux contraintes de cisaillement. Le cheveu est également un matériau élastique qui recouvre en général sa forme d'origine après avoir été soumis à une contrainte. En outre, la forme naturelle des cheveux est très variable dans le monde, en fonction des individus et de leurs origines ethniques : le cheveu peut être naturellement raide, ondulé, bouclé, frisé ou crépu, tandis que sa section, plus ou moins elliptique, a un diamètre moyen pouvant être compris entre 45 et 100 microns. Ces différences varient de manière relativement continue entre les différents peuples de la planète, mais l'on peut cependant distinguer trois grands types de cheveux dans le monde [AMB01] : les cheveux asiatiques, africains, et caucasiens.

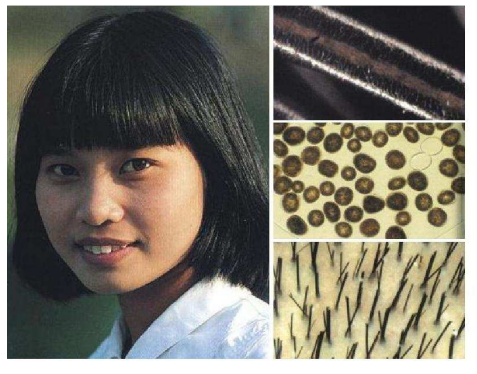

Asiatique

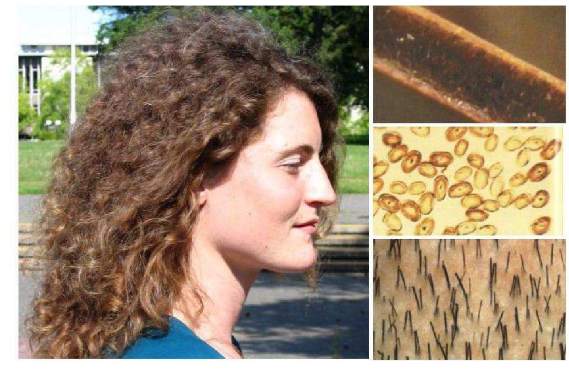

Caucasien

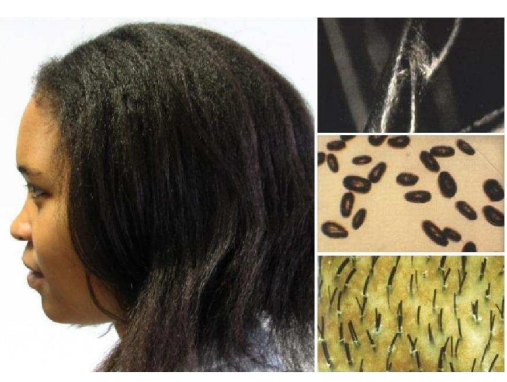

Africain

FIG. 2 - Les trois grands types de cheveux dans le monde. De gauche à droite : cheveux asiatiques, cheveux caucasiens et cheveux africains. Pour chaque catégorie est donnée une vue transversale d'un cheveu (grossi environ 400 fois), une vue des cheveux en coupe, et l'implantation des cheveux sur le cuir chevelu (vues au microscope (C) L'Oréal).

Contrairement aux cheveux asiatiques qui sont gros, réguliers, généralement droits, de section ronde et implantés perpendiculairement sur le dessus de la tête, les cheveux africains sont irréguliers, crépus, de section elliptique, et poussent de manière quasiment parallèle au cuir chevelu. Les cheveux caucasiens représentent l'intermédiaire entre les deux catégories précédentes, et peuvent avoir une forme très variable allant de raide à très frisée (voir Figure 2).

\subsection{Travaux précédents}

De nombreuses méthodes en informatique graphique synthétisent la forme finale de la chevelure à partir de surfaces ou de volumes paramétriques; l'utilisateur peut alors déformer manuellement et de manière interactive la forme de la chevelure dans l'espace en déplaçant des points de contrôle [KH00, YXYW00, KN02]. Ces techniques garantissent à l'utilisateur un bon niveau de contrôle sur la forme qu'il crée mais exigent également de sa part un important travail de modélisation pouvant parfois s'avérer fastidieux. D'autre part, une fois le modèle de coiffure réalisé, il n'est pas possible d'effectuer automatiquement des opérations de coupe ou de mouillage physiquement réalistes, car le modèle ne prend pas en compte les propriétés mécaniques de la chevelure.

D'autres approches utilisent des modèles pseudo-physiques afin d'automatiser et de rendre plus réaliste le processus de modélisation de coiffure [AUK92, HMT00, Yu01, CK05]. Dans ces approches, cependant, la géométrie fine des détails de la chevelure - tels que les boucles ou les ondulations - est ajoutée à la main ou bien de manière procédurale [Yu01, CK05]. Récemment, les méthodes à base de reconstruction d'images ont montré leur potentiel quant à leur capacité à capturer fidèlement la géométrie d'une chevelure réelle ainsi que les orientations des fibres [PBS04, WOQH05]. Cependant, comme ces méthodes ne peuvent reproduire que la partie visible de la chevelure (quasi-surface), elles sont d'une part incapables de capturer l'ensemble d'une chevelure, d'autre part les formes comportant des parties cachées, comme les boucles par exemple, posent des problèmes à la reconstruction.

Un certain nombre de travaux ont été réalisés sur la simulation physique de fils, à partir de modèles Lagrangiens [NR01, LMGC04], ou bien en utilisant le modèle de tige déformable de Cosserat régi par les équations de Kirchhoff [Pai02]. Ce dernier modèle, bien connu par la communauté de modélisation mécanique, a l'avantage de tenir compte à la fois de la déformation en courbure et en torsion d'une tige. Pai a été le premier chercheur à présenter ce modèle à la communauté d'informatique graphique [Pai02]. Dans ce travail, il propose de simu- 
ler interactivement un fil en résolvant les équations de Kirchhoff statiques de manière itérative. Notre modèle de cheveu repose également sur la description de Cosserat pour les tiges élastiques, mais contrairement à Pai, nous résolvons le problème statique grâce à une minimisation d'énergie potentielle. Cette nouvelle formulation nous permet notamment de simuler une dizaine de cheveux en temps réel, de manière stable, en prenant en compte la section elliptique du cheveu ainsi que les forces de collision pouvant s'exercer sur le modèle.

\subsection{Contributions}

Notre but est de générer de manière physiquement réaliste des formes naturelles de chevelures, à partir des propriétés structurelles et physiques du cheveu réel d'origine. Nos contributions spécifiques sont les suivantes :

1. Une amélioration du modèle de Pai [Pai02] pour la simulation physique d'un cheveu unique, grâce à une nouvelle formulation équivalente des équations de Kirchhoff dans le cas statique, basée sur une minimisation d'énergie. Cette nouvelle approche permet de prendre en compte de manière directe les forces extérieures conservatives, tout en augmentant efficacité et robustesse.

2. L'extension du modèle au traitement de cheveux bouclés et à section elliptique. Cela est en effet essentiel si l'on souhaite générer des chevelures caractéristiques de différents groupes ethniques, l'ellipticité de la section et la frisure naturelle du cheveu jouant toutes les deux un rôle prédominant dans la forme finale de la chevelure.

3. Une manière simple et directe de fournir à l'utilisateur des outils classiques de coiffure tels que le mouillage, la coupe et le séchage. En effet, grâce à notre modèle physique, ces opérations peuvent être effectuées de manière réaliste en modifiant un nombre réduit de paramètres (longueur des cheveux, rayon moyen ou module de Young). Ce nouveau modèle permet d'envisager, dans l'avenir, la conception de prototypes pour des systèmes virtuels de tests cosmétiques et de coiffure.

Nous présentons tout d'abord, en Section 2, le modèle de cheveu statique utilisé, puis expliquons le passage à l'échelle de la chevelure en Section 3. Enfin, nous validons la méthode en Section 4, avant de conclure.

\section{Simulation d'un cheveu statique}

Comme suggéré par Audoly et Pomeau [AP07], nous nous inspirons des théories de Kirchhoff et de Cosserat sur les tiges déformables pour modéliser le comportement mécanique statique d'un cheveu unique. Ce modèle de cheveu unique servira ensuite de base à la construction d'un modèle de mèche, présenté en Section 3.

\subsection{Modèle de tige de Cosserat}

Nous représentons un cheveu comme une tige inextensible, non soumise au cisaillement, et dont l'un des bords est encastré dans le cuir chevelu, l'autre étant libre. Nous choisissons de prendre une tige à section elliptique, car c'est la géométrie qui est la plus fidèle à la géométrie d'un cheveu réel. La forme de la section elliptique sera paramétrée par son excentricité ${ }^{2}$ mathématique $e$. Nous verrons dans les équations mécaniques que l'excentricité a un effet non négligeable sur la forme du cheveu.

Dans cette partie, nous considérons que le cheveu est uniquement soumis au champ de la pesanteur. La prise en compte d'autres forces extérieures dans le modèle, et notamment des forces de contact, sera abordée en Section 3.1.

Dans le modèle de tige de Cosserat [EC09], et sous les hypothèses plus restreintes de Kirchhoff dans lesquelles nous nous plaçons (tige élastique, inextensible et sans cisaillement), la configuration d'une tige est donnée par deux éléments :

- une courbe paramétrée de l'espace $\{\mathbf{r}(s), s \in[0, L]\}$, appelée courbe moyenne, décrivant la géométrie de l'axe central de la tige. On supposera dans la suite que $\mathbf{r}(s)$ est au moins de classe $\mathcal{C}^{2}$.

- une base orthonormale $\left(\mathbf{n}_{1}(s), \mathbf{n}_{2}(s), \mathbf{t}(s)\right)$ attachée en chaque point de la courbe moyenne. En général, le vecteur $\mathbf{t}$ est la tangente à la courbe $\mathbf{r}(s)$ en $s$, et les vecteurs $\mathbf{n}_{1}$ et $\mathbf{n}_{2}$ définissent le plan de la section droite locale de la tige. Dans notre cas, nous choisirons $\mathbf{n}_{1}$ et $\mathbf{n}_{2}$ comme les axes principaux de la section elliptique.

\footnotetext{
${ }^{2}$ L'excentricité d'une ellipse est définie comme le rapport de la distance du centre au foyer et la longueur du demi-grand axe. Concrètement, l'excentricité est nulle pour un cercle, et à mesure qu'elle croît, l'ellipse s'aplatit.
} 
Sous les hypothèses de Kirchhoff, une tige de Cosserat peut localement se plier autour de chacun des axes $\mathbf{n}_{1}$ et $\mathbf{n}_{2}$ (phénomène de courbure), ou bien s'enrouler autour d'elle-même (phénomène de torsion). Ceci est illustré par la Figure 3, à gauche. La courbure matérielle $\kappa_{1}$ (respectivement $\kappa_{2}$ ) quantifie la rotation locale de la tige autour de $\mathbf{n}_{1}$ (respectivement $\mathbf{n}_{2}$ ), tandis que la torsion $\tau$ caractérise la rotation locale de la tige autour de l'axe tangent $\mathbf{t}$. L'évolution spatiale le long de la tige est donnée par les équations cinématiques suivantes :

$$
\begin{aligned}
\frac{\partial \mathbf{r}}{\partial s} & =\mathbf{t} \\
\forall i \quad \frac{\partial \mathbf{u}_{\mathbf{i}}}{\partial s} & =\boldsymbol{\Omega} \wedge \mathbf{u}_{\mathbf{i}}
\end{aligned}
$$

où $\boldsymbol{\Omega}=\kappa_{1} \mathbf{n}_{\mathbf{1}}+\kappa_{2} \mathbf{n}_{\mathbf{2}}+\tau \mathbf{t}$ est le vecteur rotation instantanée de la tige, et $\mathbf{u}_{\mathbf{i}}$ représente respectivement $\mathbf{n}_{1}, \mathbf{n}_{2}, \mathbf{t}$ pour $i=1,2,3$.
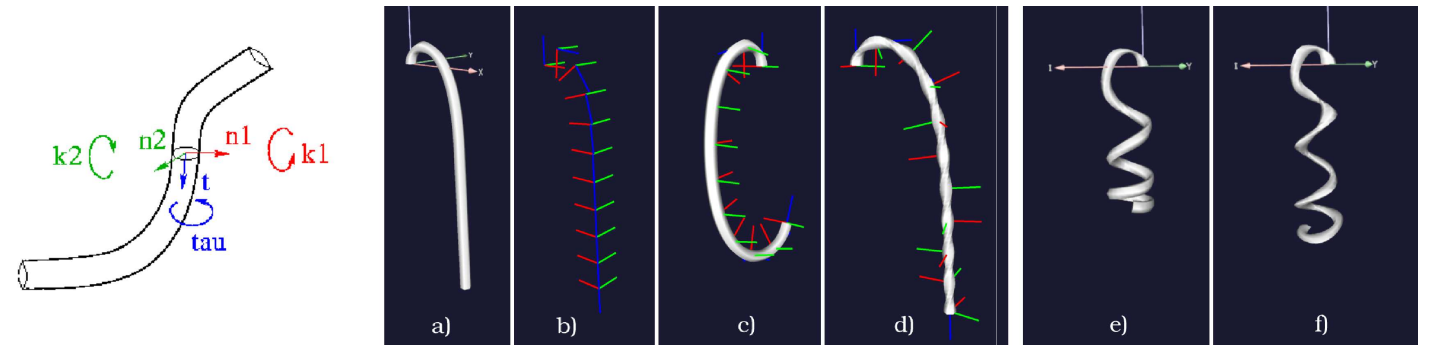

FIG. 3 - Simulation statique d'un cheveu. À gauche : une tige de Cosserat et un repère matériel; la courbure $\kappa_{1}$ (resp. $\kappa_{2}$ ) indique de combien tourne la tige autour de $n_{1}$ (resp. $n_{2}$ ), et la torsion $\tau$ indique de combien tourne la tige autour de $t$. À droite : différentes configurations d'une tige de Cosserat à l'équilibre : a) tige soumise à la gravité; b) quelques repères matériels le long de la tige; $c)$ tige présentant une courbure naturelle non nulle; d) tige enroulée sur elle-même, ie. avec une torsion naturelle non nulle ; $e)$ : tige très bouclée, de section circulaire; f) tige très bouclée, de section elliptique.

Une propriété bien connue et qui nous sera très utile pour déterminer la configuration finale d'une tige de Cosserat est alors la suivante : si $\kappa_{1}, \kappa_{2}$ et $\tau$ sont constantes le long de la tige de Cosserat (ie. indépendantes de $s$ ), alors la configuration de la tige est une hélice [AP07].

\subsection{Configuration de la tige au repos}

Comme mentionné précédemment, un cheveu peut être bouclé naturellement. Cette frisure naturelle et intrinsèque est prise en compte dans notre modèle par les courbures naturelles, notées $\kappa_{1}^{0}$ et $\kappa_{2}^{0}$, et la torsion naturelle, notée $\tau^{0}$. Ces paramètres décrivent en fait l'état de repos du système, lorsqu' aucune force extérieure n'est appliquée. Dans le cas des cheveux, il paraît raisonnable de supposer que les courbures et torsion naturelles sont à peu près constantes le long du cheveu; en effet, la frisure naturelle d'un cheveu est prédéterminée par sa racine, et on peut donc penser qu'elle reste inchangée durant toute la phase de croissance du cheveu. Précisons cependant que cette hypothèse n'est en rien une restriction du modèle. Notons également que dans ce cas, la configuration de la tige au repos est une hélice d'après la propriété énoncée précédemment.

\section{3 Énergie potentielle}

Nous cherchons les configurations à l'équilibre d'un cheveu soumis au champ gravitationnel, connaissant ses caractéristiques physiques (angle d'encastrement, longueur, rayon, excentricité de sa section elliptique, courbures et torsion naturelles). Une solution consisterait à résoudre les équations de Kirchhoff. Cette approche, suivie par Pai [Pai02], mène à un système d'équations différentielles ordinaires non linéaires avec des conditions aux limites aux deux extrémités (un bord encastré, l'autre libre). Ce type d'équations ne peut se résoudre que de manière itérative et la non unicité de la solution peut soulever des difficultés numériques. Pour éviter ces dernières, nous proposons une formulation des équations de Kirchhoff physiquement équivalente, fondée sur une minimisation d'énergie, qui s'avère plus facile à résoudre et plus fiable. 
Les configurations d'équilibre d'un système physique peuvent être obtenues par la recherche des minima de son énergie potentielle. L'énergie potentielle d'une tige de longueur $L$, soumise au champ de pesanteur, s'écrit :

$$
\mathcal{E}_{\text {hair }}=\mathcal{E}_{g}+\mathcal{E}_{e}
$$

où $\mathcal{E}_{e}$ est l'énergie élastique interne de la tige et $\mathcal{E}_{g}$ l'énergie potentielle de pesanteur. En supposant que le matériau constituant le cheveu obéit à la loi de Hooke, l'énergie potentielle élastique interne du cheveu s'écrit :

$$
\mathcal{E}_{e}=\int_{0}^{L}\left[\frac{E I_{1}}{2}\left(\kappa_{1}(s)-\kappa_{1}^{0}\right)^{2}+\frac{E I_{2}}{2}\left(\kappa_{2}(s)-\kappa_{2}^{0}\right)^{2}+\frac{\mu J}{2}\left(\tau(s)-\tau^{0}\right)^{2}\right] d s
$$

où $E$ est le module de Young (ou module d'élasticité) du cheveu, $\mu$ son module de cisaillement ${ }^{3}, I_{1}$ (respectivement $I_{2}$ ) le moment d'inertie de la section de la tige par rapport à l'axe $\mathbf{n}_{1}$ (respectivement, par rapport à l'axe $\mathbf{n}_{2}$ ), et $J$ le moment d'inertie axial de la tige. Ces moments d'inertie dépendent des rayons principaux de la section du cheveu, et donc du paramètre excentricité $e$.

L'énergie potentielle de pesanteur du cheveu $\mathcal{E}_{g}$ peut s'écrire ainsi :

$$
\mathcal{E}_{g}=\rho S g \int_{0}^{L} z(s) d s
$$

où $\rho$ est la masse volumique du cheveu, $S$ l'aire de sa section (supposée constante le long du cheveu), $g$ la valeur du champ gravitationnel et $z(s)$ la cote de l'élément $d s$ à l'abscisse curviligne $s$.

D'après l'équation (2.3), l'énergie potentielle élastique $\mathcal{E}_{e}$ d'un cheveu est minimale (et égale à zero) lorsque les courbures et torsion $\kappa_{1}, \kappa_{2}, \tau$ de la tige sont égales à leur valeur spontanée $\kappa_{1}^{0}, \kappa_{2}^{0}, \tau^{0}$. Cette tendance du cheveu à recouvrer sa forme naturelle hélicoïdale est contrebalancée par l'effet de la gravité, représenté par $\mathcal{E}_{g}$, qui consiste à tirer le cheveu vers le bas. L'énergie potentielle totale du cheveu $\mathcal{E}_{\text {hair }}$ est donc minimale lorsqu'il y a équilibre entre ces deux effets antagonistes.

\subsection{Résolution numérique}

La tige est tout d'abord subdivisée en $n$ morceaux $s_{i}$ que l'on prendra de longueur égale $d s$ par souci de simplification. Sur chaque (petit) morceau de tige $s_{i}$, on suppose que les courbures $\kappa_{i}$ et la torsion $\tau$ sont constantes. Soit $C_{n}$ le vecteur de taille $3 n$ composé des $n$ courbures $\kappa_{1}^{i}, \kappa_{2}^{i}$ et torsions $\tau^{i}$. Comme le repère initial $\mathcal{F}(0)$ est imposé par l'encastrement dans le cuir chevelu, le vecteur $C_{n}$ définit une configuration unique pour la tige. En effet, la courbe $\mathbf{r}(s)$ et le repère matériel $\mathcal{F}(s)$ peuvent se déduire de manière unique le long de la tige à partir des $\kappa_{1}^{i}, \kappa_{2}^{i}$ and $\tau^{i}$, en utilisant les équations pour une hélice sur chaque morceau.

Nous cherchons tout d'abord à trouver le vecteur $C_{n}$ qui minimise l'énergie $\mathcal{E}_{\text {hair }}$ de la tige, puis à calculer la configuration spatiale correspondante de la tige. Voici l'algorithme que nous utilisons pour ce faire :

1. L'énergie du cheveu $\mathcal{E}_{\text {hair }}$ et le vecteur $C_{n}$ sont initialisés à des valeurs arbitraires ;

2. Tant que l'énergie $\mathcal{E}_{\text {hair }}$ continue à décroître, on itère :

- Calcul de l'énergie élastique $\mathcal{E}_{e}$ par l'équation (2.3);

- Calcul formel de la configuration $(\mathbf{r}, \mathcal{F})$ de la tige, chaque morceau de la tige étant une hélice.

- Calcul de l'énergie potentielle de pesanteur $\mathcal{E}_{g}$, qui requiert l'intégration des équations cinématiques $(2.1 \mathrm{~b})$, afin de déterminer la fonction $z(s)$. En effet, $z(s)=<\mathbf{r}(s), \mathbf{e}_{\mathbf{z}}>$ avec la condition $z(0)=0$, où $\mathbf{e}_{\mathbf{z}}$ est le vecteur du repère du monde (constant) orienté verticalement. En utilisant l'équation (2.1a), on en déduit l'expression suivante pour $\mathcal{E}_{g}$ :

$$
\mathcal{E}_{g}=\rho S g \int_{0}^{L}\left(L-s^{\prime}\right)<\mathbf{t}\left(s^{\prime}\right), \mathbf{e}_{\mathbf{z}}>d s^{\prime}
$$

qui peut être évalué de manière précise en pratique.

- Minimisation de $\mathcal{E}_{\text {hair }}$.

${ }^{3}$ Le module de cisaillement $\mu$ de la tige s'exprime en fonction du module d'Young $E$ et du coefficient de Poisson $\sigma$ par : $\mu=\frac{E}{2(1+\sigma)}$ 
3. Connaissant le vecteur $C_{n}$ qui minimise $\mathcal{E}_{\text {hair }}$, on calcule la configuration finale $\{(\mathbf{r}(s), \mathcal{F}(s)), s \in[0, L]\}$ de la tige, en utilisant les équations pour les hélices.

La Figure 3 montre quelques configurations de cheveux à l'équilibre, obtenues par notre algorithme de minimisation d'énergie. Remarquons que lorsque l'on augmente l'excentricité de la section de la tige, tout en maintenant un rayon moyen constant, la régularité des boucles augmente le long de la tige.

Finalement, notre méthode est assez rapide pour traiter une dizaine de cheveux en temps réel, et une centaine en quelques secondes, chaque cheveu étant discrétisé en 15 morceaux. Ces performances satisfaisantes nous permettent d'envisager ce modèle pour la génération de coiffures naturelles complètes via une interface interactive.

\section{Modélisation d'une coiffure complète}

De même que Choe et al. [CK05], nous représentons une chevelure complète comme un ensemble de mèches, dans lequel la forme de chaque mèche est gouvernée par un cheveu directeur, et l'apparence visuelle donnée par le tracer d'un ensemble de cheveux individuels à l'intérieur du volume de la mèche à l'étape du rendu. La forme du cheveu directeur est déterminée par l'algorithme de simulation statique d'un cheveu unique présenté en section précédente, tandis que la forme des cheveux rendus est générée à partir de celle du cheveu directeur, par un processus stochastique similaire à celui utilisé dans [CK05].

\subsection{Collisions}

Afin de créer des coiffures réalistes, il est nécessaire de tenir compte des interactions se produisant entre les mèches de cheveux, la tête et le corps du personnage, ainsi qu'au sein de la chevelure. Il est en particulier indispensable de traiter les collisions entre mèches si l'on veut que les coiffures générées aient un volume adéquat.
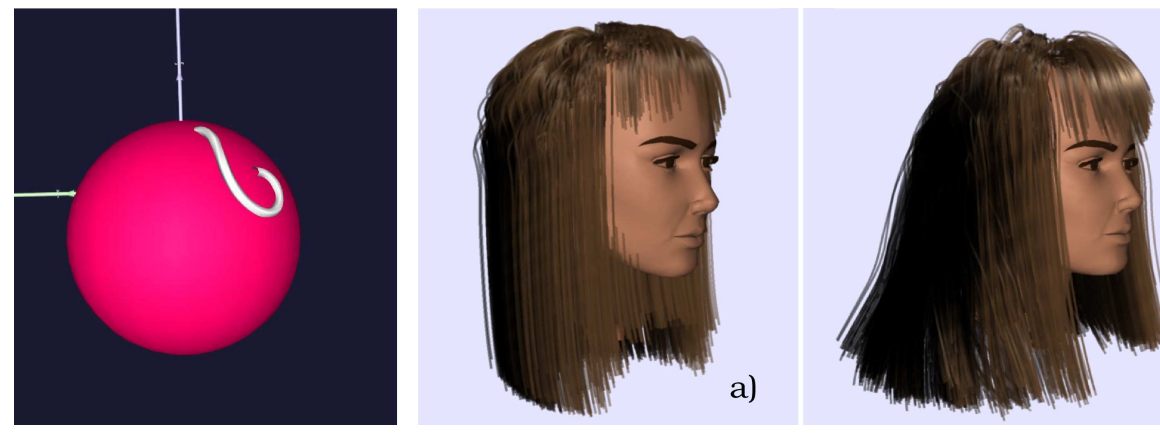

b)

FIG. 4 - À gauche : collision entre un cheveu bouclé et une sphère. À droite : comparaison entre une chevelure complète sans collisions (a), et une chevelure pour laquelle l'ensemble des collisions (entre mèches, et entre les mèches et la tête), est correctement pris en compte (b). Remarquer en particulier la différence de volume entre les deux coiffures.

Pour les collisions, nous représentons chaque mèche $w$ de manière simplifiée par un squelette, composé d'un nombre réduit de points d'échantillonnage $s_{w}^{i}$ du cheveu directeur (en pratique, ce nombre est pris égal au nombre de morceaux composant le cheveu directeur), ainsi que d'une série d'épaisseurs $r_{w}^{i}$ le long de la mèche, calculées en fonction de plusieurs facteurs tels que le nombre de cheveux composant la mèche et le niveau de frisure de la mèche. Le corps du personnage est approché par un ensemble de sphères qui servent à la fois pour la détection des collisions, et pour la réponse. Lorsqu'une collision se produit entre une mèche et une sphère du corps, nous appliquons au point de contact du squelette une force de pénalité élastique, notée $F_{c}$. L'avantage est qu'une telle force dérive d'une énergie potentielle, et donc pour tenir compte de sa contribution dans la simulation, il suffit d'ajouter son énergie correspondante $\mathcal{E}_{c}$ dans l'équation (2.2). Pour trouver la forme finale du cheveu directeur de la mèche en collision, le problème revient alors simplement à minimiser la nouvelle énergie $\mathcal{E}_{\text {hair }}$ définie ainsi :

$$
\mathcal{E}_{\text {hair }}=\mathcal{E}_{g}+\mathcal{E}_{e}+\mathcal{E}_{c}
$$

En pratique, on évalue $\mathcal{E}_{c}$ en fonction de la distance $x$ d'interpénétration entre la mèche et le corps : $\mathcal{E}_{c}=\frac{1}{2} k x^{2}$, où $k$ est un paramètre de raideur choisi arbitrairement. Comme illustré sur la Figure 4 (à gauche), cette méthode, bien que très simple, simule parfaitement les contacts entre un cheveu directeur et une sphère. 
Pour traiter les collisions entre mèches de manière efficace, nous nous sommes inspirés de l'algorithme des couches multiples d'enveloppes développé par Lee et Ko [LK01]. Le principe repose sur l'hypothèse suivante : les cheveux plantés haut sur le crâne doivent toujours recouvrir les cheveux plantés plus bas. L'idée consiste alors à créer une série d'enveloppes autour de la tête, en pelures d'oignon, et à détecter la collision de chaque cheveu avec l'enveloppe correspondant à l'altitude du cheveu (plus un cheveu est haut, plus on le teste avec une enveloppe grande). Cette méthode n'est autre que l'extension de l'algorithme de détection de collisions entre les cheveux et la tête. Bien sûr, elle ne marche que lorsque la tête reste statique et orientée verticalement, ce qui est notre cas. La Figure 4 (à droite, b)) montre qu'une chevelure complète présente un volume satisfaisant, grâce à cette approche.

\subsection{Outils d'édition de coiffure}

Cette partie décrit les outils de coiffure virtuelle dont dispose l'utilisateur pour créer des coiffures. Par rapport aux approches précédentes, notre méthode présente le grand avantage de simplifier la gestion des opérations classiques de coiffure telles que la coupe, le mouillage ou le séchage, grâce au modèle physique sous-jacent.

\subsubsection{Effet de l'eau sur les cheveux}

Si l'on veut simuler une chevelure mouillée pour des applications de coupe et de coiffure virtuelle, il est nécessaire de connaître les phénomènes physiques qui se produisent lorsque les cheveux sont en contact avec l'eau. Le cheveu est en effet perméable à l'eau, et peut absorber jusqu'à $40 \%$ de son propre poids en eau. Cette absorption d'eau cause, entre autres, un changement important des propriétés mécaniques et géométriques du cheveu : son diamètre augmente de $13 \%$ environ, sa longueur de 1\%, et son module d'élasticité décroît d'un facteur 5 [BW05], rendant le cheveu beaucoup plus déformable. Enfin, une chevelure mouillée est beaucoup moins volumineuse qu'à sec, à cause de la nature cohésive de l'eau.

Ward et al. ont proposé un modèle de chevelure tenant compte de ces propriétés [WGL04]. Mais, comme leur modèle physique est très simple (chaines masses-ressorts), et donc n'est pas paramétré par des quantités physiques adéquates, il leur faut contrôler de multiples structures pour arriver à appliquer sur la chevelure les changements physiques correspondant au passage à l'état mouillé. Au contraire, grâce à notre approche basée sur un système mécanique réaliste, mouiller une chevelure revient simplement du point de vue de la simulation à modifier quelques paramètres physiques, et à changer les forces de collisions entre mèches. L'aspect visuel des mèches est également modifié pour un rendu plausible. Concrètement, notre algorithme de mouillage est le suivant :

- Le module d'élasticité des cheveux directeurs est divisé par 5, leur rayon augmenté de 13\% et leur longueur de $1 \%$. En pratique, c'est principalement le module d'élasticité qui a un fort impact sur la forme de la mèche.

- Nous diminuons le rayon de chaque mèche d'environ $40 \%$ (d'après nous, cela correspond de manière satisfaisante à la réalité). La distance d'interpénétration $x$ dans le calcul des énergies de collision (voir Section 3.1) est réduite d'autant.

- Au niveau du rendu visuel, la couleur des cheveux fonce d'environ $20 \%$ et la diffusion de la lumière par les fibres est réduite (d'après les données expérimentales rapportées dans [BW05]).

Pour le séchage, nous considérons pour l'instant qu'il s'agit du processus inverse du mouillage, bien que ce ne soit pas tout à fait le cas dans la réalité [BW05]. Dans notre interface, un seul bouton intégrant l'ensemble de ces modifications permet à l'utilisateur de changer directement l'état de la chevelure (mouillé ou sec).
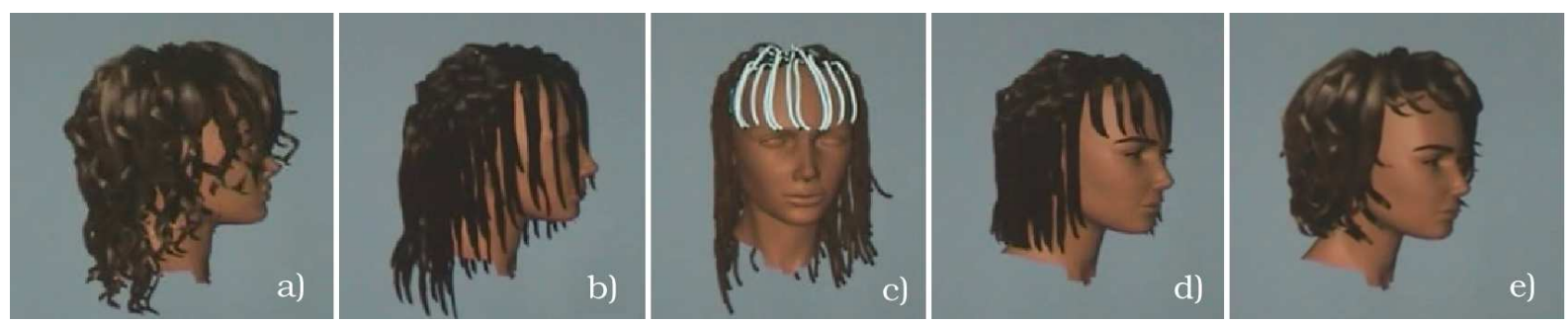

FIG. 5 - Mouillage (b), sélection par l'utilisateur des mèches pour la coupe (c), coupe finale (d) et séchage (e) d'une chevelure naturellement bouclée (a). Noter la différence de forme des mèches entre l'état mouillé et l'état sec ( $a-b$ ou d-e), causée en particulier par le changement du module d'élasticité entre les deux états. On observe également, à sec, que les mèches (sur la frange notamment) ont changé de forme du fait de la coupe : les boucles ont "remonté", comme dans la réalité. 


\subsubsection{Coupe de cheveux}

Nous considérons, pour simplifier, que la coupe de cheveux consiste à enlever la matière de la chevelure qui est située en-dessous d'un plan horizontal $z=z_{c}$. Au lieu de calculer exactement l'intersection entre le plan de coupe et chaque mèche pour en déduire la longueur finale de la mèche après la coupe, nous faisons l'approximation suivante : la longueur finale d'une mèche coupée peut être approchée par $z_{r}-z_{c}$ où $z_{r}$ est la cote de la racine de la mèche considérée. Cette approximation est raisonnable si l'on effectue la coupe sur des cheveux mouillés (donc verticaux et assez "droits"), et elle est d'autant plus correcte que les cheveux à couper sont longs (l'erreur d'approximation étant la plus importante près de la racine).

Notons que, contrairement aux approches précédentes, notre méthode de coupe est physique (i.e. la chevelure perd effectivement de la masse), et non pas purement géométrique. Ainsi, la forme de chaque boucle de la chevelure, ainsi que le volume global de cette dernière sont affectés par une coupe, comme dans la réalité (voir Figure 5).

\subsection{Rendu d'une chevelure}

Au cours de la modélisation de coiffure, le rendu des cheveux est effectué interactivement par les méthodes de Kajiya et Kay [KK89] (pour l'illumination locale) et Bertails et al. [BMC05] (pour le calcul de l'auto-ombrage). Pour le rendu final d'une coiffure, l'illumination locale des cheveux est calculée de manière plus précise par l'algorithme de Marschner et al. [MJC ${ }^{+} 03$ ]. En particulier, ce modèle tient compte des effets visuels dus à la nature elliptique de la section d'un cheveu. Ainsi, l'excentricité $e$ représente un paramètre unique de simulation, utilisé à la fois par le modèle mécanique et pour le rendu. La valeur de ce paramètre est fixée en fonction du type de cheveu considéré : pour les cheveux asiatiques, $e$ est pratiquement nul, tandis que pour les cheveux caucasiens, $e$ varie entre 0 et 0,1 , et pour les cheveux africains, $e$ varie entre 0 et 0,2 . Les modifications des paramètres de rendu effectuées pour simuler une chevelure mouillée sont données en Section 3.2.1.

\section{Validation du modèle}

\subsection{Pousse de cheveux}

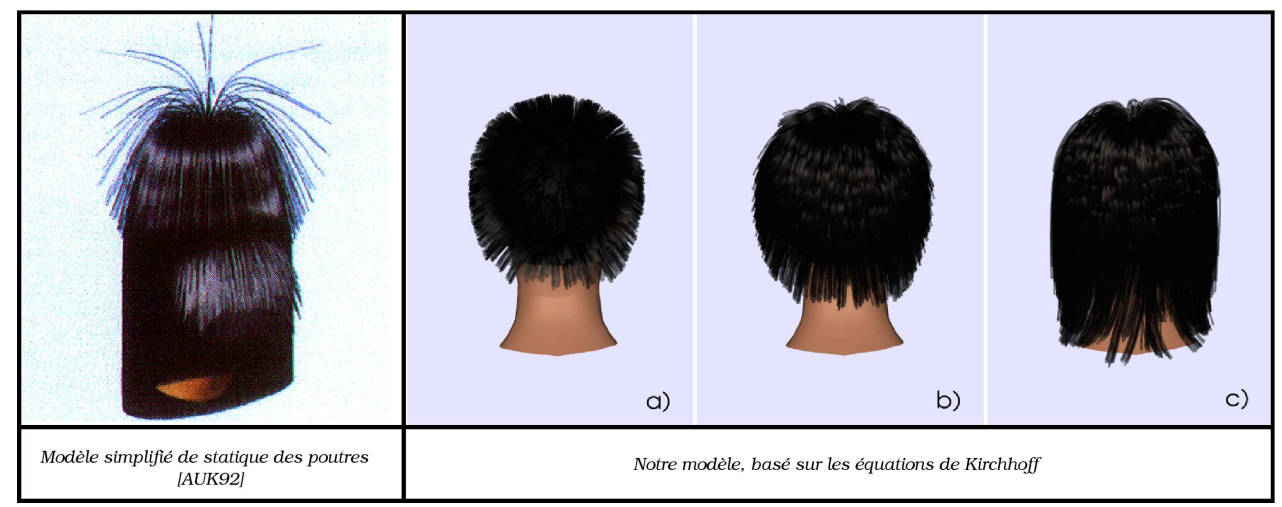

FIG. 6 - Comparaison du phénomène de pousse des cheveux entre le modèle simplifié de statique des poutres utilisé par Anjyo et al. [AUK92] (à gauche) et notre modèle basé sur les équations de Kirchhoff pour les tiges élastiques (à droite). Dans le premier cas, la gravité seule ne suffit pas à faire tomber les cheveux correctement sur la tête, et des forces supplémentaires devront être ajoutées par la suite pour obtenir une coiffure réaliste. Dans le second cas, le modèle non linéaire que nous utilisons reproduit parfaitement le phénomène se produisant lors de la pousse de cheveux: les cheveux poussent d'abord tout droit (a), puis ayant atteint une longueur seuil (b), ils se plient naturellement sous leur propre poids (b et $c$ ).

Contrairement aux nombreuses approches pseudo-physiques qui utilisent des champs de vecteurs dans l'espace 3D pour déterminer la forme statique des cheveux [HMT00, Yu01, CK05], notre méthode se sert des caractéristiques physiques des cheveux (longueur, masse volumique, module de Young, etc.) pour calculer automatiquement la forme de la chevelure sous l'effet de la gravité. La méthode d'Anjyo et al. [AUK92], qui s'est inspirée des travaux 
en résistance des matériaux sur la statique des poutres, suit un peu la même idée : dans cette approche, les cheveux se courbent naturellement sous l'effet de leur propre poids. Cependant, la méthode employée est très simplifiée (elle se base sur une équation de déformation linéaire), et n'est applicable en théorie que pour les petites déformations. Utilisée telle quelle, elle donne des résultats irréalistes (voir Figure 6), et pour générer des coiffures plausibles, elle requiert l'ajout de forces autres que la gravité (forces "coiffantes").

Au contraire, notre méthode permet de tenir compte correctement du phénomène de pousse d'un cheveu, caractérisé par le fait que, au-delà d'une certaine longueur, un cheveu même parfaitement vertical cesse de pousser droit, et plie sous l'effet de son propre poids (instabilité connue sous le nom d'elastica d'Euler).

\subsection{Génération de formes naturelles de chevelure}

La Figure 7 expose quelques résultats de coiffures que nous avons réalisées en s'inspirant de chevelures réelles, de formes diverses. Les paramètres physiques généraux utilisés dans les simulations sont fixés à leur vraie valeur, donnée par des mesures physiques sur le cheveu : $E=10 \mathrm{GPa}, \mu=0.3, \rho=1.3 \mathrm{~g} / \mathrm{cm}^{3}$ [Rob02].
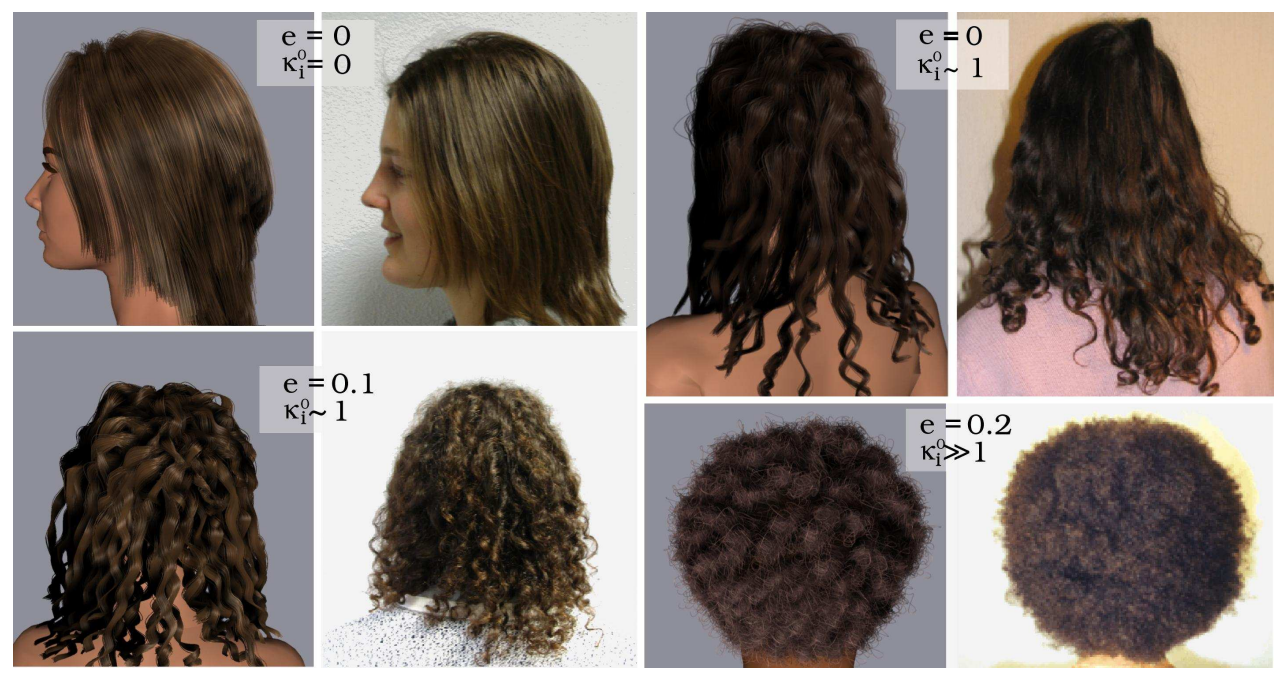

FIG. 7 - Comparaison entre des coiffures générées par notre logiciel et des coiffures réelles. À droite: images réelles de cheveux (a) raides, (b) bouclés et (c) crépus. À gauche : résultats synthétiques correspondants, générés à partir de valeur adéquates pour l'excentricité de la section du cheveu e et pour les courbures naturelles $\kappa_{i}^{0}$. Chacune des coiffures de synthèse a été réalisée à l'aide de notre logiciel en moins de 30 minutes.

Nous avons remarqué que les paramètres excentricité et courbures naturelles avaient un fort impact sur le type de boucles générées. Si la valeur des courbures naturelles correspond au degré de frisure souhaité, l'ellipticité de la section du cheveu influe sur la régularité des boucles obtenues le long du cheveu (voir Figure 7).

\section{Conclusion}

Nous avons présenté une nouvelle méthode permettant de calculer automatiquement la géométrie d'une chevelure naturelle à partir des caractéristiques structurelles, physiques et ethniques du cheveu. Comme le montrent nos résultats, cette approche permet de générer des formes de chevelures variées et fidèles à la réalité.

Actuellement, notre méthode est principalement limitée par le coût en temps de calcul nécessaire pour générer la configuration à l'équilibre d'une chevelure complète (6 secondes en moyenne sont nécessaires pour calculer une configuration à l'équilibre d'une chevelure semblable à celles de la figure 7). La limitation des performances de l'application nous empêche d'utiliser plus d'une centaine de cheveux directeurs dans notre système de modélisation de coiffure. Cependant, les résultats obtenus sont visuellement très satisfaisants avec ce nombre limité de cheveux directeurs, grâce à notre modèle procédural de mèche.

Nous étudions actuellement la prise en compte de l'angle d'encastrement des cheveux dans le cuir chevelu pour 
générer la forme finale d'une chevelure. En effet, l'implantation des cheveux a un impact assez important sur la forme d'une chevelure, surtout lorsque celle-ci est courte. Nous envisageons de plus d'intégrer au modèle quelques caractéristiques artificielles (raies par exemple) pour obtenir des styles de coiffure plus variés. Enfin, nous travaillons à l'extension de notre modèle physique pour la simulation dynamique de chevelures.

\section{Remerciements}

Merci à Steve Marschner pour nous avoir permis d'utiliser son code d'illumination locale.

\section{Références}

[AMB01] M.-C. Auzou and S. Melchior-Bonnet. Les vies du cheveu. Gallimard, 2001.

[AP07] B. Audoly and Y. Pomeau. Elasticity and Geometry : from hair curls to the nonlinear response of shells. Oxford University Press, To Appear in 2007.

[AUK92] K. Anjyo, Y. Usami, and T. Kurihara. A simple method for extracting the natural beauty of hair. In Proceedings of ACM SIGGRAPH'92, Computer Graphics Proceedings, Annual Conference Series, pages 111-120, August 1992.

[BMC05] F. Bertails, C. Ménier, and M-P. Cani. A practical self-shadowing algorithm for interactive hair animation. In Proc. Graphics Interface, May 2005.

[BW05] C. Bouillon and J. Wilkinson. The Science of Hair Care. Taylor \& Francis, New York, 2005.

[CK05] B. Choe and H-S. Ko. A statiscal wisp model and pseudophysical approaches for interactive hairstyle generation. IEEE Transactions on Visualization and Computer Graphics, 11(2), March 2005.

[EC09] E. and F. Cosserat. Théorie des corps déformables. Hermann, 1909.

[HMT00] S. Hadap and N. Magnenat-Thalmann. Interactive hair styler based on fluid flow. In Computer Animation and Simulation '00, pages 87-100, August 2000.

[KH00] C. Koh and Z. Huang. Real-time animation of human hair modeled in strips. In Computer Animation and Simulation'00, pages 101-112, September 2000.

[KK89] J. Kajiya and T. Kay. Rendering fur with three dimensional textures. In Proceedings of ACM SIGGRAPH'89, Computer Graphics Proceedings, Annual Conference Series, pages 271-280, 1989.

[KN02] T-Y. Kim and U. Neumann. Interactive multiresolution hair modeling and editing. ACM Transactions on Graphics, 21(3) :620-629, July 2002. Proceedings of ACM SIGGRAPH'02.

[LK01] D-W. Lee and H-S. Ko. Natural hairstyle modeling and animation. Graphical Models, 63(2):67-85, March 2001.

[LMGC04] J. Lenoir, P. Meseure, L. Grisoni, and C. Chaillou. A suture model for surgical simulation. 2nd International Symposium on Medical Simulation (ISMS'04), pages 105-113, june 17-18 2004.

$\left[\mathrm{MJC}^{+}\right.$03] S. Marschner, H. Jensen, M. Cammarano, S. Worley, and P. Hanrahan. Light scattering from human hair fibers. ACM Transactions on Graphics (Proceedings of the SIGGRAPH conference), 22(3) :281290, July 2003.

[NR01] O. Nocent and Y. Remion. Continuous deformation energy for dynamic material splines subje ct to finite displacements. In Eurographics Workshop in Computer Animation and Simulation, pages 87-97, Manchester, United Kingdom, sep 2001. Springer Verlag.

[Pai02] D. Pai. Strands : Interactive simulation of thin solids using cosserat models. Computer Graphics Forum, 21(3) :347-352, 2002. Proceedings of Eurographics'02.

[PBS04] S. Paris, H. Briceño, and F. Sillion. Capture of hair geometry from multiple images. ACM Transactions on Graphics (Proceedings of the SIGGRAPH conference), 2004.

[Rob02] C. Robbins. Chemical and Physical Behavior of Human Hair. 4th ed. Springer, 2002.

[WGL04] K. Ward, N. Galoppo, and M. C. Lin. Modeling hair influenced by water and styling products. In International Conference on Computer Animation and Social Agents (CASA), May 2004.

[WOQH05] Y. Wei, E. Ofek, L. Quan, and H.Y.Shum. Modeling hair from multiple views. ACM Transactions on Graphics (Proceedings of the SIGGRAPH conference), 2005.

[Yu01] Y. Yu. Modeling realistic virtual hairstyles. In Proceedings of Pacific Graphics'01, pages 295-304, October 2001.

[YXYW00] X. D. Yang, Z. Xu, J. Yang, and T. Wang. The cluster hair model. Graphics Models and Image Processing, 62(2):85-103, March 2000. 